Louisiana State University

LSU Digital Commons

4-1-2019

\title{
2900 square degree search for the optical counterpart of short gamma-ray burst GRB 180523B with the zwicky transient facility
}

\author{
Michael W. Coughlin \\ California Institute of Technology \\ Tomás Ahumada \\ University of Maryland, College Park \\ S. Bradley Cenko \\ NASA Goddard Space Flight Center \\ Virginia Cunningham \\ University of Maryland, College Park \\ Shaon Ghosh \\ University of Wisconsin-Milwaukee
}

See next page for additional authors

Follow this and additional works at: https://digitalcommons.Isu.edu/physics_astronomy_pubs

\section{Recommended Citation}

Coughlin, M., Ahumada, T., Cenko, S., Cunningham, V., Ghosh, S., Singer, L., Bellm, E., Burns, E., De, K., Goldstein, A., Golkhou, V., Kaplan, D., Kasliwal, M., Perley, D., Sollerman, J., Bagdasaryan, A., Dekany, R., Duev, D., Feeney, M., Graham, M., Hale, D., Kulkarni, S., Kupfer, T., Laher, R., Mahabal, A., Masci, F., Miller, A., Neill, J., Patterson, M., Riddle, R., Rusholme, B., Smith, R., \& Tachibana, Y. (2019). 2900 square degree search for the optical counterpart of short gamma-ray burst GRB 180523B with the zwicky transient facility. Publications of the Astronomical Society of the Pacific, 131 (998) https://doi.org/10.1088/ 1538-3873/aaff99

This Article is brought to you for free and open access by the Department of Physics \& Astronomy at LSU Digital Commons. It has been accepted for inclusion in Faculty Publications by an authorized administrator of LSU Digital Commons. For more information, please contact ir@lsu.edu. 


\section{Authors}

Michael W. Coughlin, Tomás Ahumada, S. Bradley Cenko, Virginia Cunningham, Shaon Ghosh, Leo P. Singer, Eric C. Bellm, Eric Burns, Kishalay De, Adam Goldstein, V. Zach Golkhou, David L. Kaplan, Mansi M. Kasliwal, Daniel A. Perley, Jesper Sollerman, Ashot Bagdasaryan, Richard G. Dekany, Dmitry A. Duev, Michael Feeney, Matthew J. Graham, David Hale, Shri R. Kulkarni, Thomas Kupfer, Russ R. Laher, Ashish Mahabal, Frank J. Masci, Adam A. Miller, James D. Neill, Maria T. Patterson, Reed Riddle, Ben Rusholme, Roger Smith, and Yutaro Tachibana 


\title{
2900 Square Degree Search for the Optical Counterpart of Short Gamma-Ray Burst GRB 180523B with the Zwicky Transient Facility
}

Michael W. Coughlin ${ }^{1}$ (1), Tomás Ahumada ${ }^{2}$, S. Bradley Cenko ${ }^{3,4}$, Virginia Cunningham ${ }^{2}$, Shaon Ghosh ${ }^{5}$, Leo P. Singer ${ }^{3}$, Eric C. Bellm ${ }^{6}$ (1), Eric Burns ${ }^{3}$, Kishalay De ${ }^{1}$, Adam Goldstein ${ }^{7}$, V. Zach Golkhou, ${ }^{8,9,21}$, David L. Kaplan ${ }^{5}$, Mansi M. Kasliwal ${ }^{1}$ (D), Daniel A. Perley ${ }^{10}$, Jesper Sollerman ${ }^{11}$, Ashot Bagdasaryan ${ }^{12}$, Richard G. Dekany ${ }^{12}$, Dmitry A. Duev ${ }^{1}$, Michael Feeney ${ }^{12}$, Matthew J. Graham ${ }^{13}$ (1) David Hale ${ }^{12}$, Shri R. Kulkarni ${ }^{1,12}$, Thomas Kupfer ${ }^{14,15}$, Russ R. Laher ${ }^{16}$, Ashish Mahabal ${ }^{13,17}$ (i), Frank J. Masci ${ }^{16}$ (D), Adam A. Miller ${ }^{18,19}$, James D. Neill ${ }^{1}$, Maria T Patterson ${ }^{8}$ (D), Reed Riddle ${ }^{12}$, Ben Rusholme ${ }^{16}$, Roger Smith ${ }^{12}$, Yutaro Tachibana ${ }^{20}$ (iD), and Richard Walters ${ }^{12}$

${ }^{1}$ Division of Physics, Math, and Astronomy, California Institute of Technology, Pasadena, CA 91125, USA

${ }^{2}$ Department of Astronomy, University of Maryland, College Park, MD 20742, USA

${ }^{3}$ Astrophysics Science Division, NASA Goddard Space Flight Center, Mail Code 661, Greenbelt, MD 20771, USA

${ }^{4}$ Joint Space-Science Institute, University of Maryland, College Park, MD 20742, USA

${ }^{5}$ Department of Physics, University of Wisconsin-Milwaukee, Milwaukee, WI 53211, USA

${ }^{6}$ Department of Astronomy, University of Washington, Seattle, WA 98195 , USA

${ }^{7}$ Science and Technology Institute, Universities Space Research Association, Huntsville, AL 35805, USA

${ }^{8}$ DIRAC Institute, Department of Astronomy, University of Washington, 3910 15th Avenue NE, Seattle, WA 98195, USA

${ }^{9}$ The eScience Institute, University of Washington, Seattle, WA 98195 , USA

${ }^{10}$ Astrophysics Research Institute, Liverpool John Moores University, IC2, Liverpool Science Park, 146 Brownlow Hill, Liverpool L3 5RF, UK

${ }^{11}$ The Oskar Klein Centre, Department of Astronomy, AlbaNova, SE-106 91 Stockholm, Sweden

${ }^{12}$ Caltech Optical Observatories, California Institute of Technology, Pasadena, CA 91125, USA

${ }^{13}$ Division of Physics, Mathematics and Astronomy, California Institute of Technology, Pasadena, CA 91125, USA

${ }^{14}$ Kavli Institute for Theoretical Physics, University of California, Santa Barbara, CA 93106, USA

${ }^{15}$ Department of Physics, University of California, Santa Barbara, CA 93106, USA

${ }^{16}$ IPAC, California Institute of Technology, 1200 E. California Blvd, Pasadena, CA 91125, USA

${ }^{17}$ Center for Data Driven Discovery, California Institute of Technology, Pasadena, CA 91125, USA

${ }^{18}$ Center for Interdisciplinary Exploration and Research in Astrophysics and Department of Physics and Astronomy, Northwestern University, 2145 Sheridan Road, Evanston, IL 60208, USA

${ }^{19}$ The Adler Planetarium, Chicago, IL 60605, USA

${ }^{20}$ Tokyo Institute of Technology, 2-12-1 Ookayama, Meguro, Tokyo 152-8551, Japan

Received 2018 November 19; accepted 2019 January 16; published 2019 February 20

\begin{abstract}
There is significant interest in the models for production of short gamma-ray bursts (GRBs). Until now, the number of known short GRBs with multi-wavelength afterglows has been small. While the Fermi GRB Monitor detects many GRBs relative to the Neil Gehrels Swift Observatory, the large localization regions makes the search for counterparts difficult. With the Zwicky Transient Facility (ZTF) recently achieving first light, it is now fruitful to use its combination of depth $\left(m_{\mathrm{AB}} \sim 20.6\right)$, field of view ( $\approx 47$ square degrees), and survey cadence (every $\sim 3$ days) to perform Target of Opportunity observations. We demonstrate this capability on GRB 180523B, which was recently announced by the Fermi GRB Monitor as a short GRB. ZTF imaged $\approx 2900$ square degrees of the localization region, resulting in the coverage of $61.6 \%$ of the enclosed probability over two nights to a depth of $m_{\mathrm{AB}} \sim 20.5$. We characterized 14 previously unidentified transients, and none were found to be consistent with a short GRB counterpart. This search with the ZTF shows it is an efficient camera for searching for coarsely localized short GRB and gravitational-wave counterparts, allowing for a sensitive search with minimal interruption to its nominal cadence.
\end{abstract}

Key words: gamma rays: general

Online material: color figures

\section{Introduction}

Gamma-ray bursts (GRBs), 50 years after their discovery by the Vela Satellites (Klebesadel et al. 1973), are still among the

\footnotetext{
${ }^{21}$ Moore-Sloan, WRF Innovation in Data Science, and DIRAC Fellow.
}

brightest observable objects in the universe. With their intrinsic brightness and discovery at cosmological distances (Metzger et al. 1997; Gehrels \& Mészáros 2012), they remain a mystery and an active area of research. Even their classification into the traditional "short" (SGRB) and "long" (LGRB) classes is 
subject to debate (Norris \& Bonnell 2006; Zhang \& Choi 2008; Bromberg et al. 2013). There are a variety of models for GRBs that have been developed since their discovery. For example, the "fireball" model (Wijers et al. 1997; Mészáros \& Rees 1998), where a highly relativistic jet of electron/ positron/baryon plasma is emitted by a compact central engine produced from the merger of two neutron stars (or one neutron star and one black hole), predicts production of gamma-rays and hard X-rays within the jet. In addition, an "afterglow" is produced by interaction of the jet with nearby ambient material, resulting in emission in the X-ray, optical, and radio for several days following the GRB. Later models have been produced to understand observed deviations in light curves seen from this model (Willingale et al. 2007; Cannizzo \& Gehrels 2009; Metzger et al. 2011; Duffell \& MacFadyen 2015), including slow-moving cocoons (Nagakura et al. 2014; Kasliwal et al. 2017; Lazzati et al. 2017; Mooley et al. 2017) and Gaussian structured jets (Kumar \& Granot 2003; Abbott et al. 2017; Troja et al. 2017). In the case of a compact merger, there is an additional component of highly neutron rich, unbound matter which is driven by radioactive decay of $r$-process elements that can heat the ejecta and power a thermal ultraviolet/optical/ near-infrared transient known as a kilonova (or macronova) (Lattimer \& Schramm 1974; Li \& Paczynski 1998; Metzger et al. 2010; Rosswog 2015; Kasen et al. 2017). These sources are expected to be broadly isotropic and therefore it may be possible to observe a kilonova regardless of the orientation of the system (Roberts et al. 2011).

With both its depth ( $m_{\mathrm{AB}} \sim 20.6$ in $\left.30 \mathrm{~s}\right)$, wide field of view (FOV) $(\approx 47$ square degrees), and rapid cadence (every $\sim 3$ days), the Zwicky Transient Facility (ZTF; Bellm et al. 2019; Dekany et al. 2018; Graham et al. 2018), a camera and associated observing system on the Palomar 48 inch telescope, is important for coordinated follow-up of short GRB events. The cadence in particular is important for follow-up, as the previous imaging provides significant constraints on the explosion time of any new transients. It improves significantly in FOV over the Palomar Transient Factory (Law et al. 2009; Rau et al. 2009), which had a 7.3 square degree imager capable of performing $\approx 100$ degrees squared searches (Singer et al. 2013), and the regular cadence of observations places stringent limits on the explosion time of new transients. It supplements existing systems like the Gravitational-wave Optical Transient Observer (O'Brien 2018) and Asteroid Terrestrial-impact Last Alert System (ATLAS) (Tonry et al. 2018). It uses "reference images," which are stacks of typically at least 15 images (Masci et al. 2019). These reference images are created for a fixed grid of tiles with minimal dithering, which is due to the desire to simplify the data processing for image subtraction (Bellm et al. 2019). At the time of paper submission (2018 November), about $70 \%$ of the sky visible from Palomar currently has reference image coverage available in $g$ - and $r$-bands; the exact numbers differ due to the varying number of observations in each filter. These references images are required for image differencing, which uses the ZOGY algorithm (Zackay et al. $2016)$ to identify moving and changing sources.

ZTF is part of the Global Relay of Observatories Watching Transients Happen (GROWTH) network of telescopes for rapid classification of identified candidates, whose follow-up is coordinated via a marshal developed for this purpose (Kasliwal et al. 2019). The idea of the marshal is that it allows timedomain astronomers to create filters to save sources from different discovery streams, such as ZTF, and coordinate follow-up with various queue scheduled or human operated telescopes.

GRB 180523B triggered the Fermi GRB Monitor at 18:46:28.11 (UTC) on 2018 May 23. It was classified as a GRB by the flight software and localized by the automated system which performs the automated production and distribution of the localizations. Its localization region had a $1 \sigma$ circular-equivalent statistical error of $13^{\circ}$ (including the systematic error results in a $90 \%$ probability region $\approx 6600$ square degrees). It was automatically flagged as a likely SGRB. The GRB was given a GBM trigger number, 180523782 , and the standard analysis is reported in the Fermi GBM online burst catalog (Bhat et al. 2016). The duration, calculated as the usual $T_{90}$, is $1.984 \mathrm{~s}$. While this is near the historical split time of $2 \mathrm{~s}$, modeling the GBM $T_{90}$ distribution as two log-normal functions yields a $90 \%$ probability that this GRB belongs to the short duration class. The time-integrated best fit model is a comptonized model (a power law with an exponential cutoff), which is typical for SGRBs. The peak energy is $\approx 1 \mathrm{MeV}(1150 \pm 711 \mathrm{keV})$, which is in the top quartile for SGRBs, lending additional evidence to the classification of a short hard GRB. The burst has a $64 \mathrm{~ms}$ peak flux at the 20th percentile for SGRBs and a fluence at the 40th percentile. The prompt brightness suggests the afterglow is likely to be of average luminosity (Nysewander et al. 2009).

We are interested in systematically detecting the optical afterglow of SGRBs to characterize their emission mechanisms and their hosts. This is a part of a broader Target of Opportunity (ToO) program searching for optical counterparts to neutrinos and gravitational waves as well. This program follows a Palomar Transient Factory program searching for LGRB afterglows from the Fermi GRB Monitor; eight afterglows were found (Singer et al. 2013, 2015). Only a few other LGRB afterglows have been discovered serendipitously, e.g., Stalder et al. (2017). These data will supplement the existing catalog of light curves from many other SGRBs (Berger et al. 2005, 2013; Fox et al. 2005; Levan et al. 2006; Roming et al. 2006; Soderberg et al. 2006; Zhang et al. 2007; McGlynn et al. 2008; Antonelli et al. 2009; Perley et al. 2009; Ackermann et al. 2010; Tanvir et al. 2013; Zhu et al. 2015; Fong et al. 2016; Troja et al. 2016; Abbott et al. 2017). The multi-wavelength data is essential for constraining redshifts, energetics, emission geometry, and conditions in the 


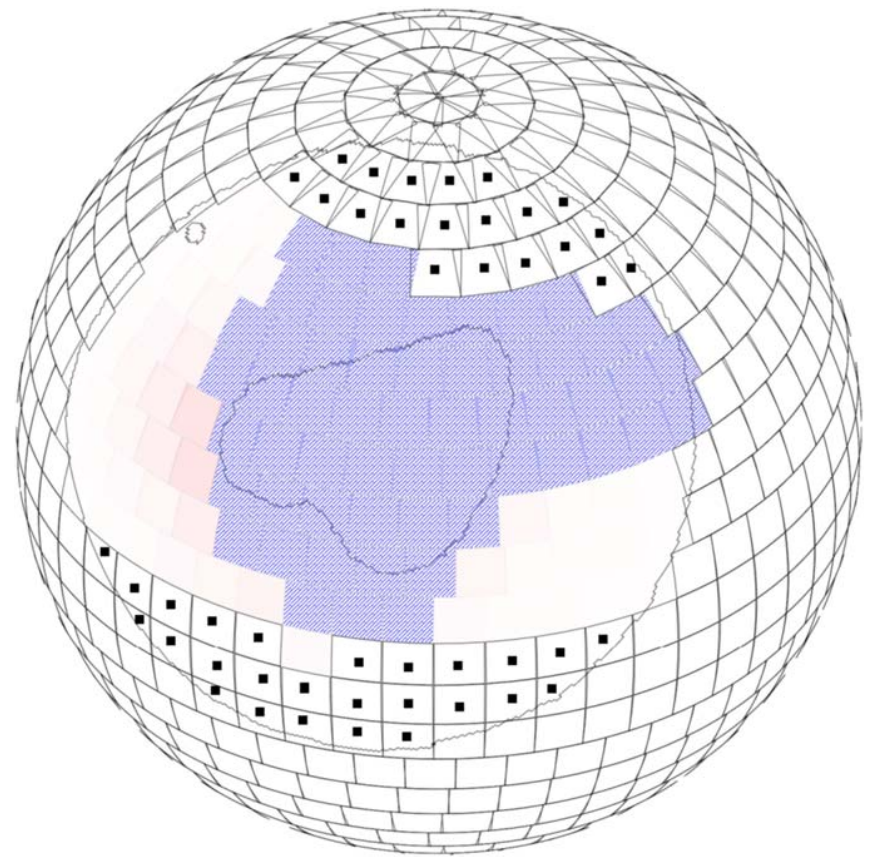

Figure 1. Target of Opportunity marshal representation of GRB 180523B scheduling. The black contours indicate the $50 \%$ and $90 \%$ credible levels. Tiles within the $90 \%$ credible interval are considered for imaging. The unshaded tiles with a single dot within the $90 \%$ credible interval do not contain reference images and therefore are not eligible to be analyzed for new transients with difference imaging. The red tiles shown contain reference images, but were not imaged due to time, airmass, or moon constraints. The checkered blue tiles shown are those scheduled to be imaged. Table 1 shows the success rate of the requested observations.

(A color version of this figure is available in the online journal.)

circumburst medium, while their locations within galaxies provide clues as to their progenitors (Fong \& Berger 2013). In this paper, we describe the $\mathrm{ToO}$ search for the optical counterpart of GRB 180523B. We describe our observing plan in Section 2. We summarize image quality information in Section 3. The identified candidates, including their follow-up, are summarized in Section 4. Section 5 summarizes our conclusions and future outlook.

\section{Observing Plan}

The observing strategy was as follows. The fields we first observed corresponded to those enclosed in the $90 \%$ localization region (see Figure 1). On the first night (2018 May 24), we planned a series of $r$-band, $g$-band, and then again $r$-band images. Each set of observations began immediately after the first; this sequence allowed for measurement of both color and fade in any transients detected, useful for their eventual classification. We note that this left more than half of the night for the regular survey mode. To determine which fields would be imaged, we removed any that would not be available at an airmass less than 3 during the portion of the night when the

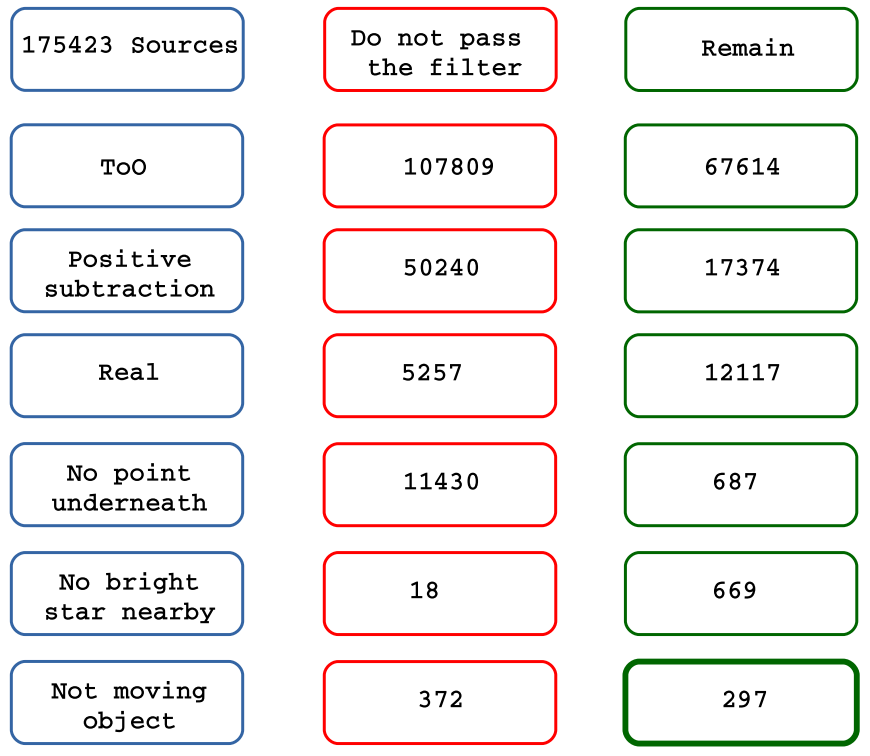

Figure 2. Flow diagram of the filtering scheme for candidate transient sources detected after difference imaging. Each step is described in Section 4.2. The number of transients that survived after all the filter steps is 297 for the night of May 25th. Objects with previous detections are still included for scanning. (A color version of this figure is available in the online journal.)

localization region was visible from Palomar. The scheduling web interface includes airmass plots as well to help human observers determine when to schedule observations for. In addition, we removed any fields without reference images in the bands of interest. From there, we scheduled $60 \mathrm{~s}$ exposures in $r$-band, $g$-band, and then $r$-band, which are twice as long as the nominal $30 \mathrm{~s}$ exposures performed in survey mode. We ordered the observations by R.A. We note that while the tiles were selected using an algorithm, they were scheduled manually by calling the on-duty astronomer. This schedule consists of an ordered list of sky locations and exposure times for the desired images. We obtained images covering $\approx 2900$ square degrees beginning at 3:51 UT on 2018 May 24, which is about $9.1 \mathrm{hr}$ after the burst trigger time. This delay was due to the fact that the SGRB alert came during the day at Palomar, and 3:51 UT was the start of nightly observations.

On the second night, we took a series of $r$-band and $g$-band exposures in the same fields as the night before. This time, $75 \mathrm{~s}$ exposures were scheduled for the same time as the night before in order to be more sensitive and catch any potentially fading counterparts. In total, we used $2: 16 \mathrm{hr}$ on the first night and $2: 43 \mathrm{hr}$ on the second night. For comparison, ZTF observed for about $7: 50 \mathrm{hr}$ those nights, and so we used $\approx 25 \%$ of the available observation time.

The ZTF FOV is comprised of 16 CCDs, each composed of 4 quadrants, placed in a $4 \times 4$ orthogonal grid, with about $12 \%$ of the nominal tile area lost to gaps between the detectors (known as chip gaps). The generation of the observation 
Table 1

Success Rates for the Requested ZTF Observation Fields Over Each Night

\begin{tabular}{lcccc}
\hline \hline Date & Fields & Fields & Fields \\
Requested & Observed & Processed & $\begin{array}{c}\text { Total Quadrant } \\
\text { Success Rate }\end{array}$ \\
\hline May 24 & 108 & 106 & 105 & $88 \%$ \\
May 25 & 95 & 94 & 94 & $98 \%$ \\
\hline
\end{tabular}

Note. The percentage of fields processed includes those with any quadrant in that field is processed.

schedule initially ignored the presence of the chip gaps. The total source-localization probability enclosed on 2018 May 24, accounting for the loss of probability due to the chip gaps, is $61.6 \%$. In the second night of observations (2018 May 25), where two observation windows in a single night was used, the total sky-localization probability enclosed in the observed fields was $68.4 \%$. The slightly greater containment of probability is due to the different airmass constraint requirements used in the first observation, where three observation windows were required. We also note that the order of the tiles by probability does not change with the inclusion of the chip gaps.

In addition to candidates that had the expected number of exposures over the two nights ( 5 if they were in a $g$ and $r$ field, 3 in just $r, 2$ in just $g$ ), some objects appeared in more than one field. In some of those cases, the objects were near the edges of the chips and were not found in those observations. There were also some objects for which there were not the expected number of exposures (only 1 in $g$, etc.). Once again, some of these candidates fall in chip gaps, which move from image to image.

\section{Image Quality}

Successful analysis of a requested ZTF observation is a twostep process. Requested fields must successfully pass through both the ZTF robotic-observing program and also through the image-processing pipeline at the Infrared Processing and Analysis Center (IPAC). Therefore, the "success rate" is the percentage of quandrants with requested observations were processed.

We show the success rate in the entire image processing pipeline in Table 1. 108 fields (with 6912 total quadrants), were requested for observation on the first night (2018 May 24). Of those 108 fields, 105 were successfully processed by IPAC. Some quadrants fail the processing due to bad seeing $\left(>5^{\prime \prime}\right)$. $12 \%$ of the quadrants failed processing for reasons of this type. 95 fields (6080 total quadrants) were requested on the second night (2018 May 25). Of those 95 fields, 94 were successfully processed by IPAC, with only $2 \%$ of quadrants failing processing. As weather conditions are the dominant contribution to missing quadrants, there is not much gain to be expected. The success rate over the course of both nights is $92.8 \%$.

We can estimate the loss of probability due to these quadrant failures. Assuming that the scales in which the sky-localization changes is much larger than the quadrant size, we can estimate the total loss of probability enclosed as $7.4 \%$ and $4.9 \%$ on night 1 and 2 respectively. The $5 \sigma$ median depth of the observations over the two nights is $m_{\mathrm{AB}}>20.3$ in $r$-band and $m_{\mathrm{AB}}>20.6$ in $g$-band.

\section{Candidates}

\subsection{Detection and Filtering}

Once the observations are taken and processed by the IPAC pipeline, alerts known as AVRO packets are created for each object (Masci et al. 2019; Patterson et al. 2019). These alerts contain information about the transient, including its magnitude, proximity to other sources and its previous history of detections among other metrics. The GROWTH collaboration has developed a framework that can handle the large number of alerts that ZTF produces in order to select the objects that are potentially interesting. Moreover, as each group within the GROWTH collaboration has a particular scientific focus, the language provides a tool to filter, discriminate and retrieve object of interest (Kasliwal et al. 2019).

In particular, for looking for optical counterparts of SGRBs, the filter restricted candidates to those with the following properties (see Figure 2):

1. Part of the ToO. The candidates must belong to the fields related to the ToO observation of GRB 180523B.

2. Positive subtraction. As the transients from ZTF images are detected by comparing reference images to the most recent image, it is required that the subtraction yields a positive difference in order to be considered a valid candidate. This is in contrast to negative subtractions, which are sources that decreased in brightness since the reference images were taken.

3. It is real. The GROWTH collaboration has developed a Real-Bogus index (Mahabal et al. 2019) trained on common image artifacts, including hot pixels and ghosts from bright stars. The index is the result of a random forest classifier and its value was restricted to $>0.15$, the optimal to determine whether the source is a product of an artifact or if it is real.

4. No point source underneath. The coordinates of the source should not coincide with a point source (Tachibana Miller 2018). This helps to rule out stellar variable sources or extragalactic transient/variable sources that are at small offsets from their hosts.

5. Two detections. The object should have at least two detections during the night; asteroids, other solar system 
objects, and cosmic rays should not have a previous detection.

6. Far from a bright star. To avoid any kind of artifacts due to bright nearby source, including diffraction spikes or ghosts, the candidate is rejected if it is in the vicinity $\left(20^{\prime \prime}\right)$ of a bright star $\left(m_{\mathrm{AB}}<15\right)$.

\subsection{Scanning and Selection}

During each night, the data are filtered, and the results are shown in the marshal for each scientific program. The interface shows the annotations retrieved from the filter, including color, cross-matches to external catalogs and positioning of the object on the chip and focal plane, as well as the historical light-curve of the object and subtracted images. This allows an on-duty astronomer to visually inspect the results of the marshal filter and proceed with a more refined candidate selection by saving the most interesting candidates into the project's report. The interface allows individuals to cross-match the candidates with a number of databases in order to rule out candidates based on the available data. For example, it is possible to rule out a candidate based on WISE colors (Wright et al. 2010), since active galactic nuclei and quasars lay in a particular locus in the WISE color-space (Stern et al. 2012). Likewise, a candidate can be ruled out if it has an ATLAS (Tonry et al. 2018) detection registered before the SGRB alert. While we do not explicitly use the $g$ - and $r$-band colors in the filtering, they are also potentially useful for classifying objects.

A number of candidates in the GROWTH report page presented a pattern of consecutive detection and non-detection in very short periods of time, thus requiring further investigation; these were determined to be artifacts from the ghosts of bright stars. In addition, we do not consider sources that were first detected by ZTF prior to the time of the SGRB. This resulted in 29 sources, 15 of which were artifacts; different scenarios can cause the presence of a bogus source, such as a nearby bright source or a bad pixel in the CCD. Thus, for GRB $180523 \mathrm{~B}$ the final candidate selection has 14 objects, whose properties are noted in Table 2; 11 of these have been followedup, as described in the following subsection.

\subsection{Follow-up and Results}

For the candidates that met the requirements, we requested additional photometry and spectroscopy. However, the observability of some of the transients was airmass constrained or required observations soon after sunset. This resulted in three candidates not being observed; these objects are noted at the bottom of Table 2 .

The GROWTH collaboration has access to a number of facilities to follow-up the candidates. Particularly, photometric data were obtained using the Kitt Peak EMCCD Demonstrator (KPED) on the Kitt Peak 84 inch telescope (Coughlin et al. 2019).
This instrument has the ability to take a set of short exposures and to modify its gain within a range, but the conventional long exposure-fixed gain mode was used for this follow-up. The photometric data taken with KPED was dark subtracted and flat field calibrated, and the magnitudes were determined using Source Extractor. The spectroscopic data were obtained using both the SED Machine (Blagorodnova et al. 2018) on the Palomar 60 inch (P60) telescope and the Double Spectrograph on the Palomar 200 inch (P200) telescope.

The classification for the candidates that had spectra was rapid and resulted in four new supernova Ia discovered. In Table 2, there is a summary of the data of the candidates. As previously mentioned, most of the candidates set very early in the night, reaching airmasses of 2 at the beginning of the observing run. Three of these candidates were not observable by follow-up facilities due to airmass constraints. However, seven of these objects were imaged with KPED a few weeks after their discovery. None of the sources had faded completely, indicating that they are not related to a SGRB.

Models and previous SGRBs detected have characterized the evolution of these kind of transients as rapidly fading sources. Thus our follow-up can discriminate between SGRBs and other transients by taking the difference between the magnitude at the discovery and the magnitude of the follow-up, as well as by examining the light curve or the spectrum if available. After having observed a significant portion of the probability region and classifying 11 of the 14 remaining transients without finding any potentially viable counterpart for the SGRB, it is possible to compare the search sensitivity, both in terms of depth and timescale, to expected counterpart properties. In Figure 3, we show where the median limits fall relative to known afterglows. Over $61.6 \%$ of the localization, we detected no afterglow emission to a depth of $m_{\mathrm{AB}}>20.3$ in $r$-band from 0.6 to 2 days after the merger. This comparison shows that future follow-ups would benefit both from a more rapid response (although in this case, the follow-up was restricted by the day time as opposed to technical issues), and taking longer exposures, resulting in deeper observations (unless the SGRB is in the local universe, as will be the case for the gravitational-wave counterpart searches). Earlier observations would not only allow for observations of objects when they are brighter, but also allow for easier identification of rapidly fading afterglows, especially when near the sensitivity limit for the telescope.

\section{Conclusions}

Our analysis demonstrates the feasibility of large scale TOO coverage of large sky areas with ZTF. Although we did not find the optical counterpart to the short GRB, the significant coverage of the sky localization region gives confidence in success for future events. In particular, the lack of unrelated sources, enabled by high cadence pre-burst limits from the regular ZTF survey, makes follow-up of thousands of square 
Table 2

Follow-up for the Candidates

\begin{tabular}{|c|c|c|c|c|c|c|}
\hline Candidate & Coordinates & Magnitude at Discovery & Date of Last Observation & Data Available & Classification & $\Delta m / \Delta t$ \\
\hline ZTF18aawozzj & $12: 31: 09.02+57: 35: 01.8$ & $g=20.2$ & June 9 & P200+DBSP Spectrum & SN Ia at $z=0.095$ & $\ldots$ \\
\hline ZTF18aawnbgg & $10: 40: 54.05+23: 44: 43.3$ & $r=19.88$ & June 9 & P200+DBSP Spectrum & SN Ia at $z=0.13$ & $\cdots$ \\
\hline ZTF18aawmvbj & $10: 12: 41.17+21: 24: 55.5$ & $r=19.75$ & June 9 & P200+DBSP Spectrum & SN Ia at $z=0.14$ & $\ldots$ \\
\hline ZTF18aawcwsx & $10: 40: 33.46+47: 02: 24.4$ & $r=19.84$ & June 5 & P60+SEDM Spectrum & SN Ia at $z=0.09$ & $\ldots$ \\
\hline ZTF18aawnbkw & $10: 38: 47.66+26: 18: 51.8$ & $r=19.91$ & June 12 & KPED $r=20.01$ & $\ldots$ & $-0.1 \mathrm{mag} / 20$ days \\
\hline ZTF18aawmqwo & $09: 52: 06.90+47: 18: 34.8$ & $r=19.98$ & June 21 & KPED $r=19.96$ & $\ldots$ & $0.02 \mathrm{mag} / 20$ days \\
\hline ZTF18aawmkik & $08: 51: 11.45+13: 13: 16.7$ & $r=19.04$ & June 12 & KPED $r=20.6$ & $\ldots$ & $-1.56 \mathrm{mag} / 20$ days \\
\hline ZTF18aawnmlm & $11: 03: 11.38+42: 07: 29.9$ & $r=20.12$ & June 19 & KPED $r=20.2$ & $\ldots$ & $-0.08 \mathrm{mag} / 18$ days \\
\hline ZTF18aauhzav & $10: 59: 29.32+44: 10: 02.7$ & $r=19.97$ & June 19 & KPED $r=21.4$ & $\ldots$ & $-0.1 \mathrm{mag} / 20$ days \\
\hline ZTF18aavrhqs & $11: 58: 09.57+63: 45: 34.6$ & $r=19.99$ & June 21 & KPED $r=21.4$ & $\cdots$ & $-1.41 \mathrm{mag} / 21$ days \\
\hline ZTF18aawmwwk & $10: 35: 26.51+65: 22: 34.3$ & $r=19.9$ & June 21 & KPED $r=19.8$ & $\ldots$ & $+0.1 \mathrm{mag} / 21$ days \\
\hline ZTF18aawwbwm & $08: 16: 44.98+35: 34: 13.1$ & $r=19.79$ & Not observable & $\ldots$ & $\ldots$ & $\ldots$ \\
\hline ZTF18aawmjru & $08: 39: 11.39+44: 01: 53.6$ & $r=18.43$ & Not observable & $\ldots$ & $\ldots$ & $\cdots$ \\
\hline ZTF18aawmigr & $08: 48: 01.76+29: 13: 51.9$ & $r=19.63$ & Not observable & $\ldots$ & $\ldots$ & $\ldots$ \\
\hline
\end{tabular}

Note. $\Delta m / \Delta t$ measures the fade in magnitudes between the first observation with ZTF and the final observation with the follow-up telescopes. For objects with this metric, the lack of rapid measured fading indicates they are unlikely to be associated with the SGRB. 

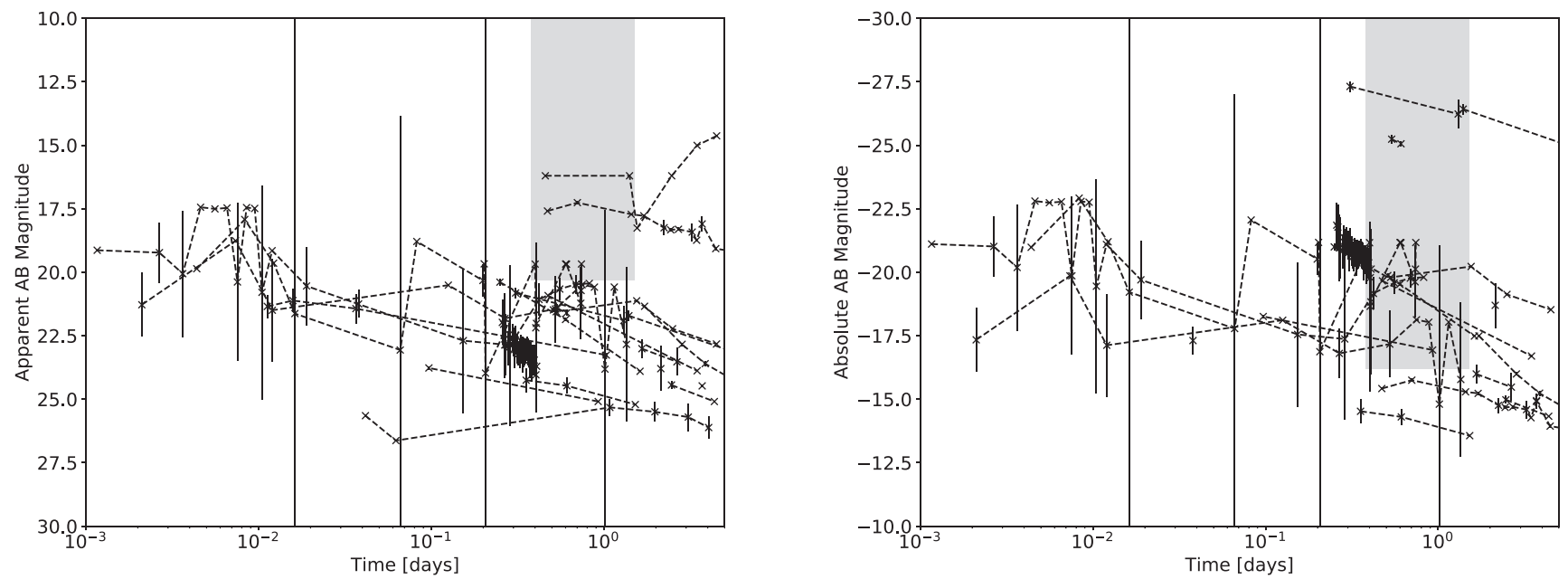

Figure 3. Light curves for known SGRBs (in the observer frame) with ZTF limits for comparison. The data are a collection of events with measured redshift from Fong et al. (2015). The ZTF search occupies the gray region in the magnitude vs. time after the trigger diagram. On the left, we plot apparent magnitude, and on the right, absolute magnitude, where we have taken a fiducial distance of $200 \mathrm{Mpc}$ for the SGRB, consistent with the LIGO/Virgo detection horizon (Abbott et al. 2018). This distance was used to transform the ZTF apparent magnitude limits into absolute magnitudes, as well as a revised distance for the SGRBs used in the sample.

degrees possible. This is a significant improvement upon the intermediate Palomar Transient Factory searches for optical counterparts to long GRBs, where for example, a 71 degrees squared search for GRB 130702A was performed in 10 fields, yielding 43 candidates (Singer et al. 2013). It also serves as a path-finder for optical follow-up for future gravitational-wave events, which is important after the significant success of GW170817, the first joint GW-EM detection (Alexander et al. 2017; Chornock et al. 2017; Cowperthwaite et al. 2017; Drout et al. 2017; Evans et al. 2017; Haggard et al. 2017; Hallinan et al. 2017; Kasliwal et al. 2017; Kilpatrick et al. 2017; Margutti et al. 2017; McCully et al. 2017; Nicholl et al. 2017; Pian et al. 2017; Shappee et al. 2017; Smartt et al. 2017; Troja et al. 2017; Utsumi et al. 2017). Some future short GRB detections are likely to yield kilonovae as well (Ascenzi et al. 2018), which enable joint gravitational-wave and electromagnetic studies (Coughlin et al. 2018a, 2018b). Our results show that for kilonovae produced from GW170817, which has a peak absolute magnitude of about $m_{\mathrm{AB}} \sim-16$, would be detectable to about $200 \mathrm{Mpc}$, consistent with the LIGO/Virgo detection horizon (Abbott et al. 2018). While the coverage of 2900 square degrees yielded a coverage of $\approx 62 \%$ of the probability region, this areal coverage would be more than sufficient to cover greater than $90 \%$ of the probability region for most future gravitational-wave events (Abbott et al. 2018). We checked that when estimating the distribution of the probability content in sky areas of similar size to this skymap using example gravitational-wave sky maps, on average about $13 \%-15 \%$ of the sky area is lost due to chip-gaps.

Going forward, we will be prioritizing minimizing the time between the notice of the short GRB and the imaging by ZTF.
These observations were scheduled by hand (Coughlin et al. 2018d), and this scheduling has now been fully automated and has been demonstrated on the most recent SGRBs that we have targeted (Cenko et al. 2018; Coughlin et al. 2018e, 2018f, 2018g). Also, there is now a filter for objects without previous detections. In addition, the scheduling will be done differently, emphasizing images of higher probability fields while also accounting for rising and setting fields and the need to make repeated exposures, potentially in different filters, to maximize the probability of counterpart detection (Coughlin et al. 2018c).

Based on observations obtained with the Samuel Oschin Telescope 48 inch and the 60 inch Telescope at the Palomar Observatory as part of the Zwicky Transient Facility project. Major funding has been provided by the U.S National Science Foundation under grant No. AST-1440341 and by the ZTF partner institutions: the California Institute of Technology, the Oskar Klein Centre, the Weizmann Institute of Science, the University of Maryland, the University of Washington, Deutsches Elektronen-Synchrotron, the University of Wisconsin-Milwaukee, and the TANGO Program of the University System of Taiwan. M.C. is supported by the David and Ellen Lee Postdoctoral Fellowship at the California Institute of Technology. S.G. is supported by the National Science Foundation with NSF Award PHY-1607585.

\section{ORCID iDs}

Michael W. Coughlin (16 https://orcid.org/0000-00028262-2924

Eric C. Bellm ๑ https://orcid.org/0000-0001-8018-5348

Mansi M. Kasliwal (1) https://orcid.org/0000-0002-5619-4938 
Matthew J. Graham (i) https://orcid.org/0000-0002-3168-0139

Ashish Mahabal, (1) https://orcid.org/0000-0003-2242-0244

Frank J. Masci $\odot$ https://orcid.org/0000-0002-8532-9395

Maria T Patterson (i) https://orcid.org/0000-0002-4753-3387

Yutaro Tachibana $\mathbb{1}$ https://orcid.org/0000-0001-6584-6945

\section{References}

Abbott, B. P., Abbott, R., Abbott, T. D., et al. 2017, ApJL, 848, L13 Abbott, B. P., Abbott, R., Abbott, T. D., et al. 2018, LRR, 21, 3 Ackermann, M., Asano, K., Atwood, W. B., et al. 2010, ApJ, 716, 1178 Alexander, K. D., Berger, E., Fong, W., et al. 2017, ApJL, 848, L21 Antonelli, L. A., D'Avanzo, P., Perna, R., et al. 2009, A\&A, 507, L45 Ascenzi, S., Coughlin, M. W., Dietrich, T., et al. 2018, arXiv:1811.05506 Bellm, E., Kulkarni, S. R., Graham, M. J., et al. 2019, PASP, 131, 018002 Berger, E., Fong, W., \& Chornock, R. 2013, ApJL, 774, L23

Berger, E., Price, P. A., Cenko, S. B., et al. 2005, Natur, 438, 988 Bhat, P. N., Meegan, C. A., von Kienlin, A., et al. 2016, ApJS, 223, 28 Blagorodnova, N., Neill, J. D., Walters, R., et al. 2018, PASP, 130, 035003 Bromberg, O., Nakar, E., Piran, T., \& Sari, R. 2013, ApJ, 764, 179 Cannizzo, J. K., \& Gehrels, N. 2009, ApJ, 700, 1047

Cenko, S. B., Coughlin, M. W., Ghosh, G., et al. 2018, GCN, 22969 Chornock, R., Berger, E., Kasen, D., et al. 2017, ApJL, 848, L19 Coughlin, M. W., Ahumada, T., Cenko, S. B., et al. 2018g, GCN, 23379 Coughlin, M. W., Cenko, S. B., Ahumada, T., et al. 2018f, GCN, 23324 Coughlin, M. W., Dekany, R. G., Duev, D. A., et al. 2019, arXiv:1901.04625 Coughlin, M. W., Dietrich, T., Doctor, Z., et al. 2018a, MNRAS, 480, 3871 Coughlin, M. W., Dietrich, T., Margalit, B., \& Metzger, B. D. 2018b, arXiv: 1812.04803

Coughlin, M. W., Singer, L. P., Ahumada, T., et al. 2018e, GCN, 22871 Coughlin, M. W., Singer, L. P., Cenko, S. B., et al. 2018d, GCN, 22739 Coughlin, M. W., Tao, D., Chan, M. L., et al. 2018c, MNRAS, 478, 692 Cowperthwaite, P. S., Berger, E., Villar, V. A., et al. 2017, ApJL, 848, L17 Dekany, R., Smith, R., Riddle, R., et al. 2018, PASP, submitted Drout, M. R., Piro, A. L., Shappee, B. J., et al. 2017, Sci, 358, 1570 Duffell, P. C., \& MacFadyen, A. I. 2015, ApJ, 806, 205

Evans, P. A., Cenko, S. B., Kennea, J. A., et al. 2017, Sci, 358, 1565 Fong, W., \& Berger, E. 2013, ApJ, 776, 18

Fong, W., Berger, E., Margutti, R., \& Zauderer, B. A. 2015, ApJ, 815, 102 Fong, W., Margutti, R., Chornock, R., et al. 2016, ApJ, 833, 151 Fox, D. B., Frail, D. A., Price, P. A., et al. 2005, Natur, 437, 845 Gehrels, N., \& Mészáros, P. 2012, Sci, 337, 932

Graham, J. M., Kulkarni, S. R., Bellm, E. C., et al. 2018, PASP, submitted Haggard, D., Nynka, M., Ruan, J. J., et al. 2017, ApJL, 848, L25 Hallinan, G., Corsi, A., Mooley, K. P., et al. 2017, Sci, 358, 1579 Kasen, D., Metzger, B., Barnes, J., Quataert, E., \& Ramirez-Ruiz, E. 2017, Natur, 551, 80

Kasliwal, M. M., Canella, C., Bagdasaryan, A., et al. 2019, PASP, 131, 038003 Kasliwal, M. M., Nakar, E., Singer, L. P., et al. 2017, Sci, 358, 1559 Kilpatrick, C. D., Foley, R. J., Kasen, D., et al. 2017, Sci, 358, 1583 Klebesadel, R. W., Strong, I. B., \& Olson, R. A. 1973, ApJL, 182, L85
Kumar, P., \& Granot, J. 2003, ApJ, 591, 1075

Lattimer, J. M., \& Schramm, D. N. 1974, ApJL, 192, L145

Law, N. M., Kulkarni, S. R., Dekany, R. G., et al. 2009, PASP, 121, 1395

Lazzati, D., López-Cámara, D., Cantiello, M., et al. 2017, ApJL, 848, L6

Levan, A. J., Tanvir, N. R., Fruchter, A. S., et al. 2006, ApJL, 648, L9

Li, L.-X., \& Paczynski, B. 1998, ApJL, 507, L59

Mahabal, A., Rebbapragada, U., Walters, R., et al. 2019, PASP, 131, 038002

Margutti, R., Berger, E., Fong, W., et al. 2017, ApJL, 848, L20

Masci, F. J., Laher, R. R., Rusholme, B., et al. 2019, PASP, 131, 018003

McCully, C., Hiramatsu, D., Howell, D. A., et al. 2017, ApJL, 848, L32

McGlynn, S., Foley, S., McBreen, S., et al. 2008, A\&A, 486, 405

Mészáros, P., \& Rees, M. J. 1998, ApJL, 502, L105

Metzger, B. D., Giannios, D., Thompson, T. A., Bucciantini, N., \& Quataert, E. 2011, MNRAS, 413, 2031

Metzger, B. D., Martínez-Pinedo, G., Darbha, S., et al. 2010, MNRAS, 406, 2650

Metzger, M., Djorgovski, S., Kulkarni, S., et al. 1997, Natur, 387, 878

Mooley, K. P., Nakar, E., Hotokezaka, K., et al. 2017, Natur, 554, 207

Nagakura, H., Hotokezaka, K., Sekiguchi, Y., Shibata, M., \& Ioka, K. 2014, ApJL, 784, L28

Nicholl, M., Berger, E., Kasen, D., et al. 2017, ApJL, 848, L18

Norris, J. P., \& Bonnell, J. T. 2006, ApJ, 643, 266

Nysewander, M., Fruchter, A., \& Pe'Er, A. 2009, ApJ, 701, 824

O'Brien, P. 2018, in COSPAR Meeting, Vol. 42, 42nd COSPAR Scientific Assembly, 18

Patterson, M. T., Bellm, E. C., Rusholme, B., et al. 2019, PASP, 131, 018001

Perley, D. A., Metzger, B. D., Granot, J., et al. 2009, ApJ, 696, 1871

Pian, E., D'Avanzo, P., Benetti, S., et al. 2017, Natur, 551, 67

Rau, A., Kulkarni, S. R., Law, N. M., et al. 2009, PASP, 121, 1334

Roberts, L. F., Kasen, D., Lee, W. H., \& Ramirez-Ruiz, E. 2011, ApJL, 736, L21

Roming, P. W. A., Berk, D. V., Palshin, V., et al. 2006, ApJ, 651, 985

Rosswog, S. 2015, IJMPD, 24, 1530012

Shappee, B. J., Simon, J. D., Drout, M. R., et al. 2017, Sci, 358, 1574

Singer, L. P., Cenko, S. B., Kasliwal, M. M., et al. 2013, ApJL, 776, L34

Singer, L. P., Kasliwal, M. M., Cenko, S. B., et al. 2015, ApJ, 806, 52

Smartt, S. J., Chen, T. W, Jerkstrand, A., et al. 2017, Natur, 551, 75

Soderberg, A. M., Berger, E., Kasliwal, M. M., et al. 2006, ApJ, 650, 261

Stalder, B., Tonry, J., Smartt, S. J., et al. 2017, ApJ, 850, 149

Stern, D., Assef, R. J., Benford, D. J., et al. 2012, ApJ, 753, 30

Tachibana Miller, Tachibana, Y., \& Miller, A. A. 2018, PASP, 130, 128001

Tanvir, N. R., Levan, A. J., Fruchter, A. S., et al. 2013, Natur, 500, 547

Tonry, J. L., Denneau, L., Heinze, A. N., et al. 2018, PASP, 130, 064505

Troja, E., Piro, L., van Eerten, H., et al. 2017, Natur, 551, 71

Troja, E., Sakamoto, T., Cenko, S. B., et al. 2016, ApJ, 827, 102

Utsumi, Y., Tanaka, M., Tominaga, N., et al. 2017, PASJ, 69, 101

Wijers, R. A. M. J., Rees, M. J., \& Mészáros, P. 1997, MNRAS, 288, L51

Willingale, R., O’Brien, P. T., Osborne, J. P., et al. 2007, ApJ, 662, 1093

Wright, E. L., Eisenhardt, P. R. M., Mainzer, A. K., et al. 2010, AJ, 140, 1868

Zackay, B., Ofek, E. O., \& Gal-Yam, A. 2016, ApJ, 830, 27

Zhang, B., Zhang, B.-B., Liang, E.-W., et al. 2007, ApJL, 655, L25

Zhang, Z.-B., \& Choi, C.-S. 2008, A\&A, 484, 293

Zhu, B., Zhang, F., Zhang, S., et al. 2015, A\&A, 576, A71 